\title{
Development of an All-in-One Phantom and Scintillator Radiation Sensor for Real-Time Monitoring of Source Position and Dose Distribution in High-Dose-Rate Brachytherapy
}

\author{
Sang Hun Shin, Wook Jae Yoo, Kyoung Won Jang, \\ Seunghyun Cho, ${ }^{1}$ Kum Bae Kim, ${ }^{2}$ and Bongsoo Lee $^{3^{*}}$ \\ School of Biomedical Engineering, BK21 Plus Research Institute of Biomedical Engineering, Konkuk University, \\ 268 Chungwon-daero, Chungju-si, Chungcheongbuk-do, 380-701, Korea \\ ${ }^{1}$ Department of Organic Materials \& Fiber Engineering, College of Engineering, Soongsil University, \\ Seoul 156-743, Korea \\ ${ }^{2}$ Department of Radiation Oncology, Korea Institute of Radiological and Medical Science, \\ Seoul 139-706, Korea \\ ${ }^{3}$ School of Energy Systems Engineering, Chung-Ang University, \\ Heukseok-dong, Dongjak-gu, Seoul, 156-756, Korea \\ (Received March 1, 2015; accepted February 26, 2016)
}

Keywords: radiation sensor, HDR brachytherapy, dose distribution, position, real-time monitoring

In this study, we developed an all-in-one phantom and scintillator radiation sensor (PSRS) with plastic scintillating fibers which can measure the real-time position and the dose distribution of Ir192 as a radioactive source employed in high-dose-rate (HDR) brachytherapy. As experimental results, we simultaneously obtained information on the shape and position of the Ir-192 as well as the dose distribution. The all-in-one PSRS system has many dosimetric advantages, such as realtime monitoring, wide dose-response range, high spatial resolution, nearly water-equivalence for high-energy photons, and the ability to measure two-dimensional dose distribution.

\section{Introduction}

The main challenge in high-dose-rate (HDR) brachytherapy is to deliver a prescribed radiation dose to a tumor while limiting the dose delivered to surrounding normal organs and tissues. In HDR brachytherapy, the iridium-192 (Ir-192) source as a gamma-emitter, with an initial radioactivity of approximately $370 \mathrm{GBq}(10 \mathrm{Ci})$ is moved and placed along catheters or applicators inserted within the treatment site during a defined period of time. The positioning and the displacement of an Ir192 source are controlled by a remote after-loader. Here, the source positions and dwell times are determined to meet the requirements for the prescribed dose. ${ }^{(1-3)}$ Therefore, it is very important to exactly measure the real-time position and the dose distribution of the Ir-192 source in after-loading HDR brachytherapy. Unfortunately, however, existing conventional methods (e.g., film based HDR brachytherapy dosimetry) cannot be used to measure the position of Ir-192 source in real time.

${ }^{*}$ Corresponding author: e-mail: bslee@naver.com 
Dose measurements of the gamma sources employed in brachytherapy are usually performed using a radiochromic film. However, the dose measurements are usually carried out using solid water or water-equivalent plastic materials because the radiochromic film may not be immersed in water. Furthermore, the radiation sensitivities of radiochromic films are different from those of tissue, and thus correction factors are needed to be applied.

To plan personalized treatment for a set of defined points to be treated, X-ray films or computed tomography (CT) images are also used in conventional HDR brachytherapy dosimetry. This dosimetry does not take into account the unique size and the shape of a tumor, or the location of an organ at risk (OAR). ${ }^{(4)}$ Accordingly, this may lead to tumor under-dosage and OAR overdosage, which can increase the probability of cancer recurrence and the incidence of side-effects, respectively. Success or failure of HDR brachytherapy depends on the exact position of the radioactive source which is used to deliver radiation to cancer cells.

In this study, we fabricated an all-in-one phantom and scintillator radiation sensor (PSRS) to measure the real-time position and the dose distribution of an Ir-192 source. The proposed PSRS offers many dosimetric advantages, such as a fast real-time response capability, wide dose response range, acceptable spatial resolution, nearly water-equivalence for high-energy photons, ability to measure two dimensional dose distributions, and convenient use for brachytherapy dosimetry.

\section{Materials and Methods}

For HDR brachytherapy dosimetry, an all-in-one PSRS was fabricated using organic scintillators and polymethylmethacrylate (PMMA) phantoms. As a sensitive material for the all-in-one PSRS, plastic scintillating fiber (BCF-20, Saint-Gobain Ceramic \& Plastics) was used to generate the scintillation light signal. BCF-20 has a core/single-clad structure with a diameter of $2 \mathrm{~mm}$. To fabricate an organic scintillator, the core of BCF-20 was synthesized with polystyrene (PS) and fluorescent dopants and the cladding material was made of PMMA. An organic scintillator is also made of low-atomic-number materials and has a low density. Therefore, an organic scintillator has nearly water-equivalent characteristics as a sensing element to measure ionizing radiation. ${ }^{(8-11)}$ The refractive indices of the core and the cladding are 1.60 and 1.49 , respectively, and the numerical aperture (NA) is approximately 0.58. The decay time and the emission peak of BCF-20 are $2.7 \mathrm{~ns}$ and $492 \mathrm{~nm}$, respectively. Table 1 shows the physical properties of BCF-20 used in this study.

Figure 1 shows the fabricated all-in-one PSRS which was composed of the plastic optical fibers and the PMMA phantoms. The plastic scintillating fibers (Nos. 1-8) of different lengths were arranged circularly in the PMMA phantom to measure the source position, and the others (Nos. 9-19) with identical lengths of $40 \mathrm{~mm}$ were arranged diagonally to measure the dose distribution, as shown in Fig. 1. Here, the length of the plastic scintillating fibers (Nos. 1-8), which are installed in a circle, increased from 5 to $40 \mathrm{~mm}$ with intervals of $5 \mathrm{~mm}$, and each scintillating fiber was spaced $4 \mathrm{~mm}$ apart.

Table 1

Physical properties of commercially available BCF-20.

\begin{tabular}{cccccccc}
\hline $\begin{array}{c}\text { Organic } \\
\text { scintillator }\end{array}$ & $\begin{array}{c}\text { Emission } \\
\text { color }\end{array}$ & $\begin{array}{c}\text { Emission } \\
\text { peak }(\mathrm{nm})\end{array}$ & $\begin{array}{c}\text { Decay time } \\
(\mathrm{ns})\end{array}$ & $\begin{array}{c}\text { 1/e length } \\
(\mathrm{m})\end{array}$ & $\begin{array}{c}\text { Number of photons } \\
\text { per Mev }\end{array}$ & $\begin{array}{c}\text { Refractive } \\
\text { index }\end{array}$ & $\begin{array}{c}\text { Density } \\
\left(\mathrm{g} / \mathrm{cm}^{3}\right)\end{array}$ \\
\hline BCF-20 & Green & 492 & 2.7 & $>3.5$ & $\sim 8000$ & 1.6 & 10.5 \\
\hline
\end{tabular}



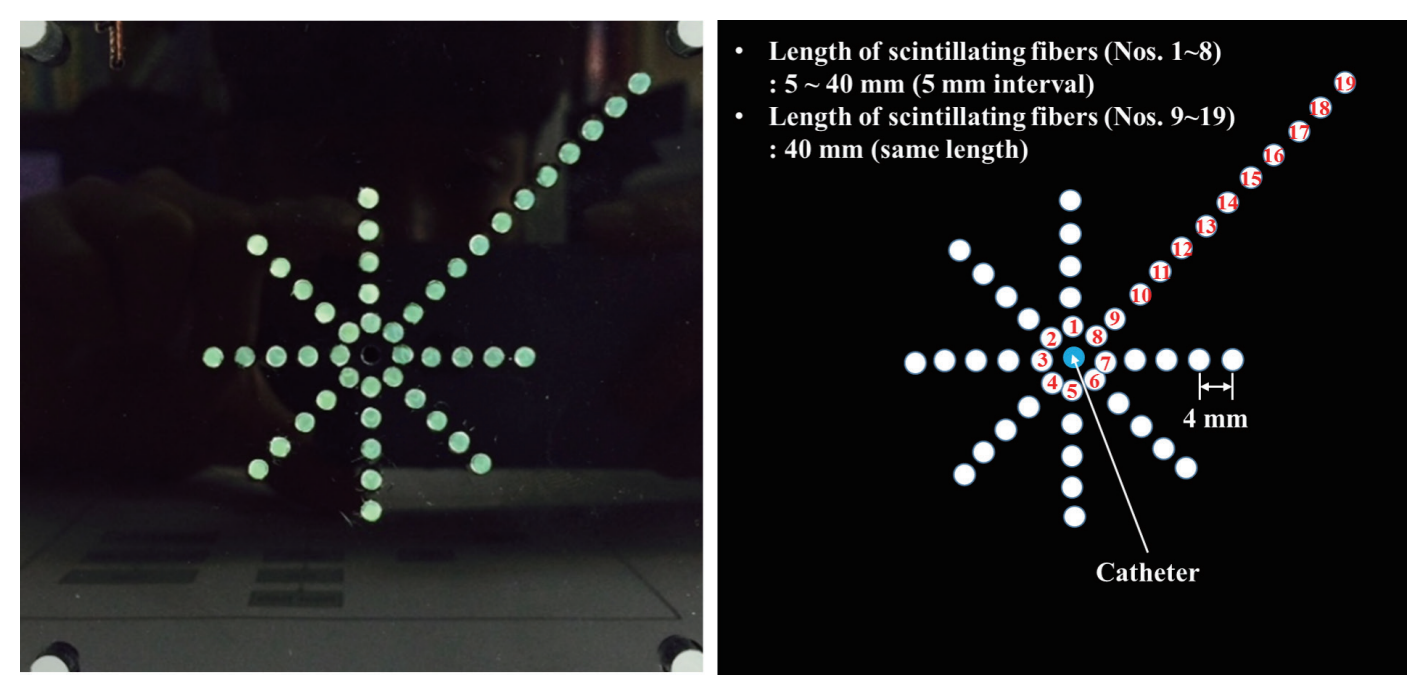

Fig. 1. (Color online) Arrangement of the plastic scintillating fibers in the all-in-one PSRS.

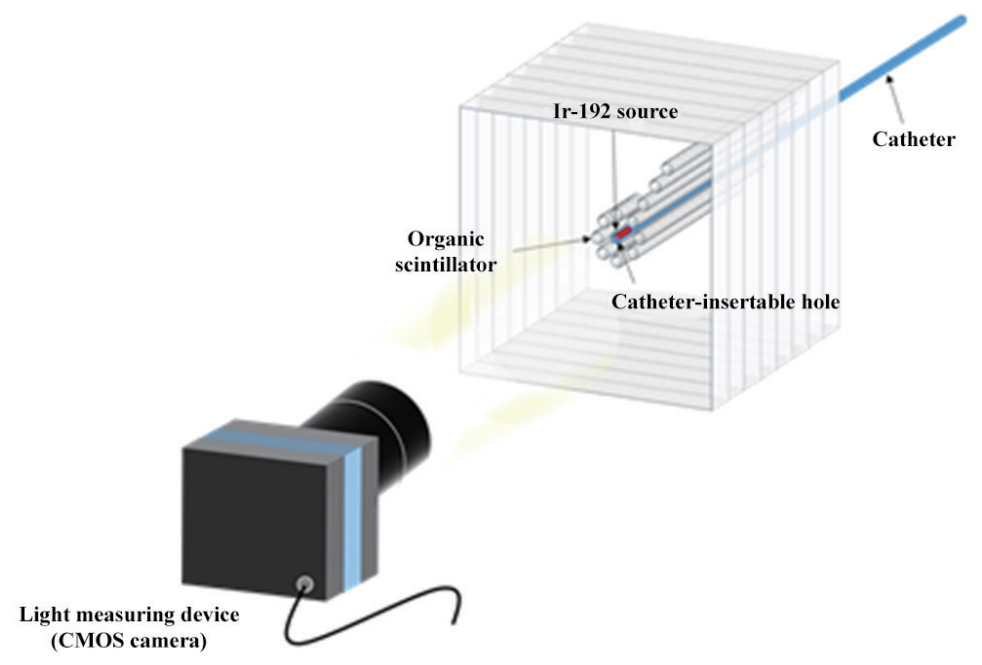

Fig. 2. (Color online) Experimental setup for measuring scintillation light using the proposed all-in-one PSRS system.

Figure 2 illustrates the experimental setup for measuring scintillation light using the all-inone PSRS, a complementary metal-oxide semiconductor (CMOS) camera, and an Ir-192 HDR brachytherapy source. Gamma-rays with an energy range from 50 to $800 \mathrm{keV}$ and two peaks at 316 and $468 \mathrm{keV}$ were emitted from the Ir-192 source with a radioactivity of $10.76 \mathrm{Ci}$. The diameter of this brachytherapy source was $0.6 \mathrm{~mm}$, and its length was $3.5 \mathrm{~mm}$. The mean energy and half-life of Ir-192 are $370 \mathrm{keV}$ and $74.2 \mathrm{~d}$, respectively. Scintillation light signals generated from the plastic scintillating fibers in the all-in-one PSRS were simultaneously measured by the CMOS camera 
(acA3800-14uc, BASLER). The image sensor of the camera consisted of a number of square pixels with dimensions of $1.67 \times 1.67 \mu \mathrm{m}^{2}$; its sensor size was $6.44 \times 4.62 \mathrm{~mm}^{2}$. The maximum resolution of the CMOS camera was $3856 \times 2764$.

\section{Results and Discussion}

Real-time images of the Ir-192 source were acquired using the proposed all-in-one PSRS system. Figure 3 shows the screen captures of the 12 scintillation images of an Ir-192 source at a series of consecutive dwell positions. While changing source position in $5 \mathrm{~mm}$ steps, we took images of the scintillation light signals using the CMOS camera. The scintillation images of allin-one PSRS contain the scintillation light signals emitted from each plastic scintillating fiber (i.e., BCF-20) to measure the real-time position of the Ir-192 source.

Figures 4 and 5 show the measurements of scintillation light signals according to the source position and the distance between each plastic scintillating fiber and the Ir-192 source. In this test, we simultaneously obtained information on the shape and the position of the Ir-192 source as well as the dose distribution. As can be seen in Figs. 3 and 4, the position of the Ir-192 source pellet can be clearly identified by analyzing the brightness (i.e., light intensity) of each scintillation light signal emitted from the circularly arranged plastic scintillating fibers (Nos. 1-8) with different lengths. As shown in Fig. 5, the intensities of each scintillation light signal emitted from the diagonally arranged plastic scintillating fibers (Nos. 9-19) with identical lengths decreased as the distance increased.
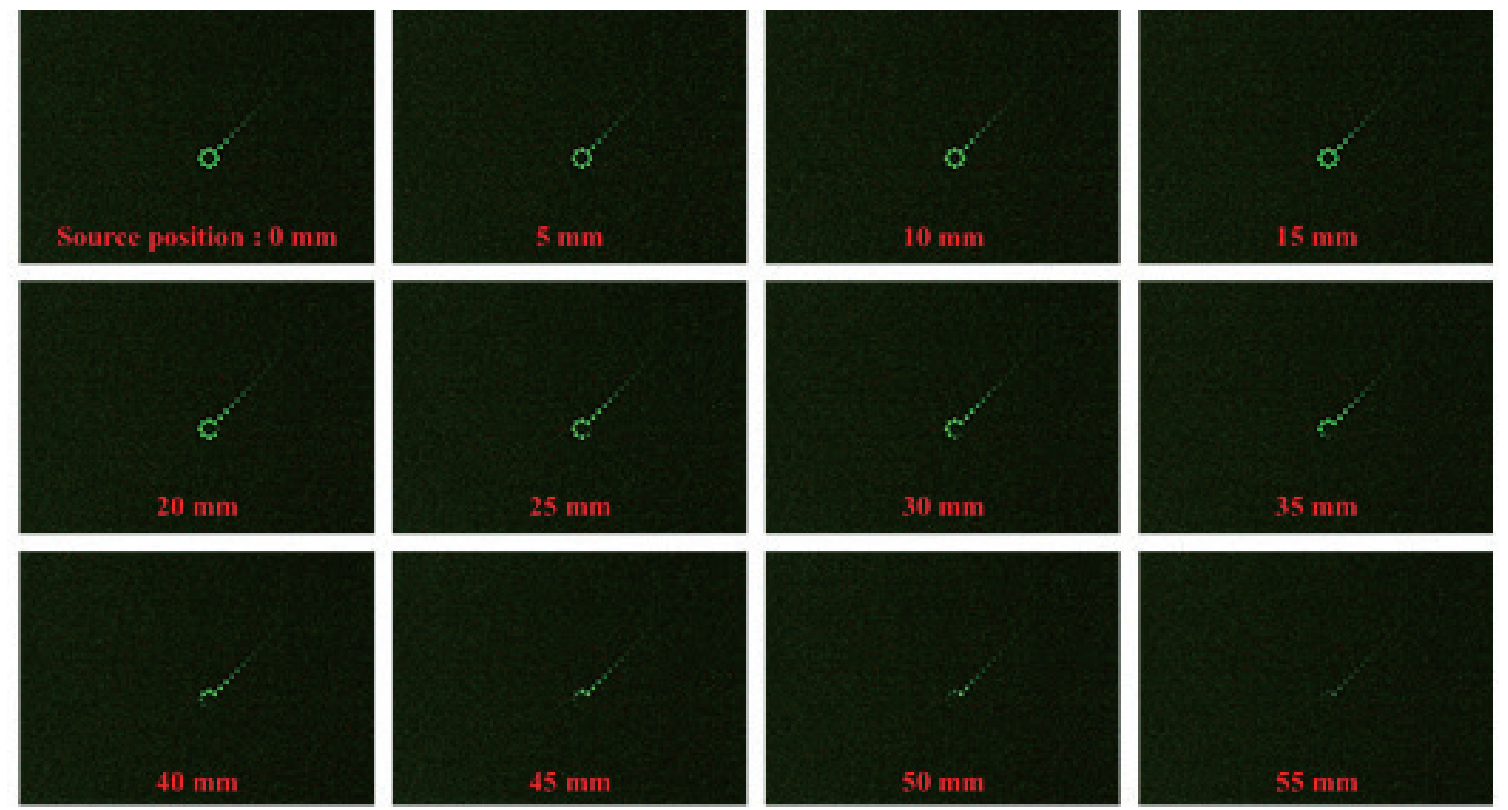

Fig. 3. (Color online) Screen captures of the 12 scintillation images of an Ir-192 source at a series of consecutive dwell positions. 


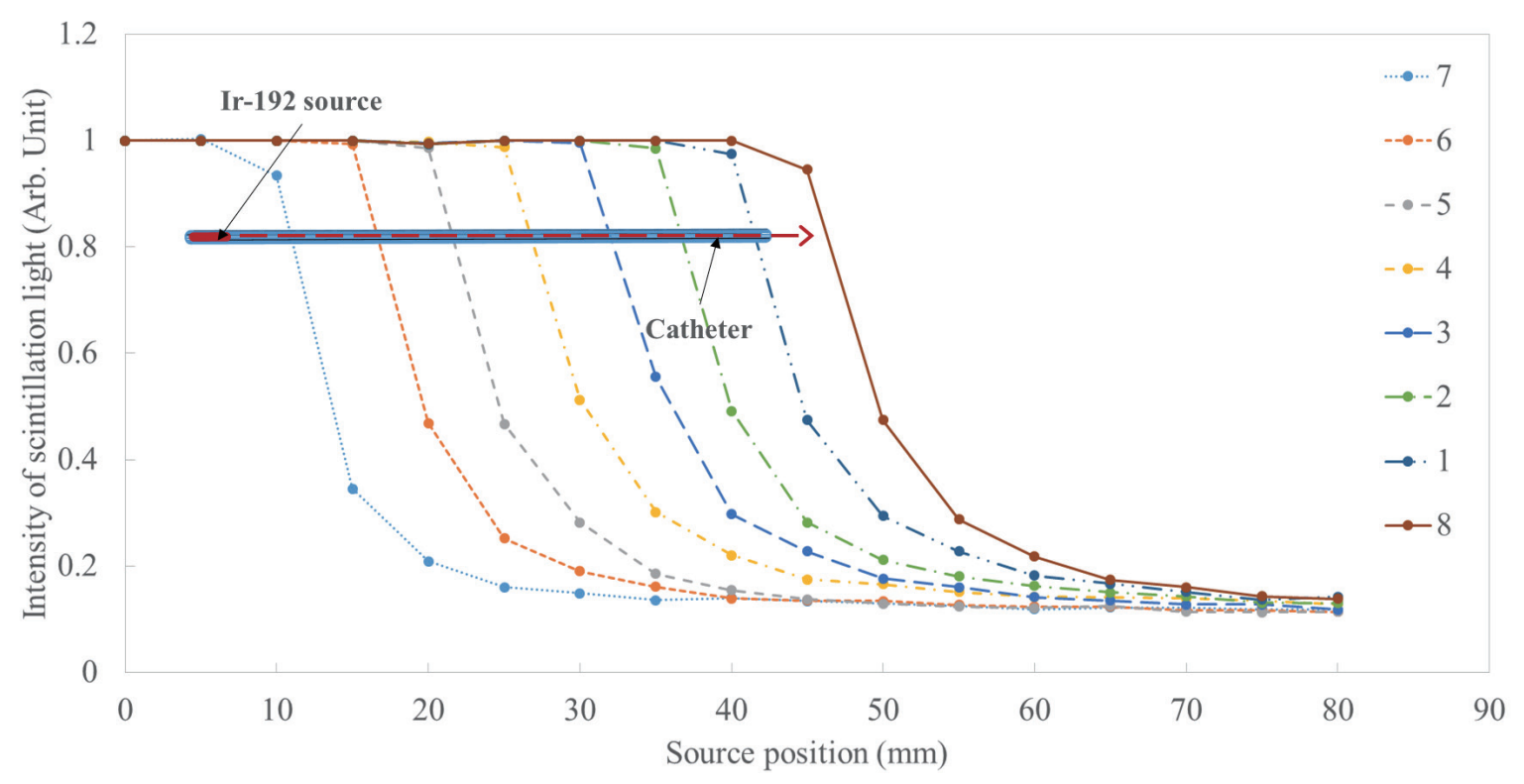

Fig. 4. (Color online) Real-time monitoring of source position using the all-in-one PSRS according to the dwell position of an Ir-192 source.

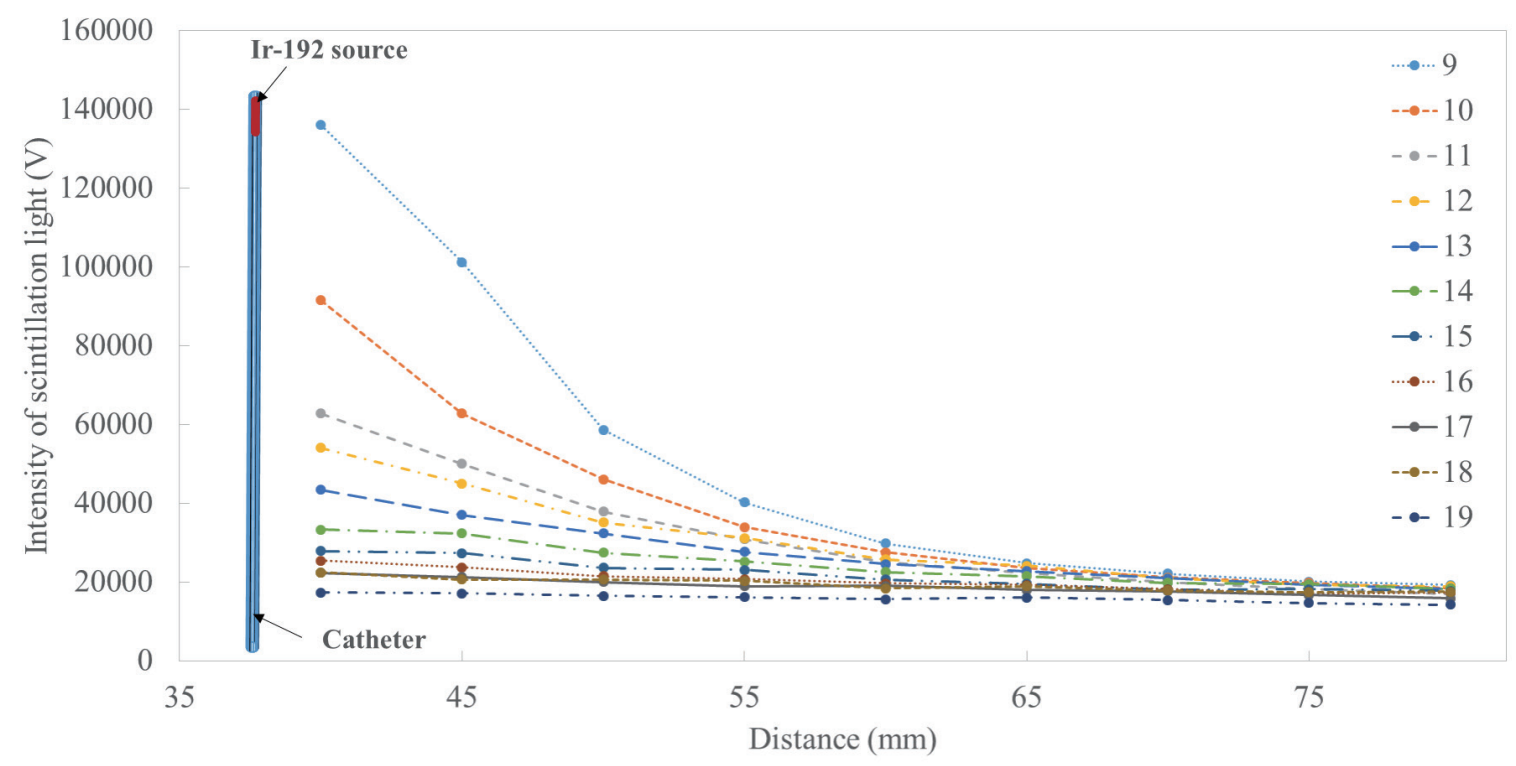

Fig. 5. (Color online) Measurements of dose distribution using the PSRS according to the distance between each plastic scintillating fiber and the Ir-192 source. 


\section{Conclusions}

We fabricated a novel all-in-one PSRS using plastic scintillating fibers and the PMMA phantoms. In this study, the scintillation images were obtained using a CMOS camera according to the position of an Ir-192 HDR brachytherapy source. In conclusion, the proposed monitoring system has a sufficient dynamic range and spatial resolution to image a small Ir-192 source under most clinical conditions. Real-time monitoring of source position using the high-resolution all-inone PSRS facilitates in vivo source tracking for the verification of the source location and the dwell times. It is expected that an all-in-one PSRS can be used in HDR brachytherapy dosimetry due to its many advantages, including high spatial resolution, real-time monitoring, ease of use, and waterequivalence. Further studies will be carried out to fabricate a newly designed all-in-one PSRS using plastic scintillating fibers with a diameter of less than $0.5 \mathrm{~mm}$ to measure real-time dose distributions with a high spatial resolution in clinical HDR brachytherapy. We will also measure the absorbed dose using a conventional ion chamber and then compare the dose values and the intensity of the scintillation signals measured using the proposed PSRS.

\section{Acknowledgements}

This research was supported by Basic Science Research Program through the National Research Foundation of Korea (NRF) funded by the Ministry of Science, ICT and future Planning (No. 2014002620). Also, this work was supported by the Nuclear Safety Research Program through the Korea Foundation Of Nuclear Safety (KOFONS), granted financial resource from the Nuclear Safety and Security Commission (NSSC), Republic of Korea (Grant No. 1403017-0114-SB110).

\section{References}

1 S. J. Goetsch, F. H. Attix, D. W. Pearson, and B. R. Thomadsen: Med. Phys. 18 (1991) 462.

2 N. P. Patel, B. Majumdar, V. Vijiyan, and P. K. Hota: J Cancer Res. Ther. 1 (2005) 213.

3 M. B. Podgorsak, L. A. DeWerd, and B. R. Paliwal: Med. Phys. 20 (1993) 1257.

4 C. C. Popescu, I. Olivotto, and V. V. Patenaude: Med. Dosim. 31 (2006) 283.

5 P. A. Jursinic and C. J. Yahnke: Med. Phys. 38 (2011) 5432.

6 L. Ludemann, C. Wybranski, M. Seidensticker, K. Mohnike, S. Kropf, P. Wust, and J. Ricke: Radiat. Oncol. 6 (2011) 1 .

7 E. L. Seymour, S. J. Downes, G. B. Fogarty, M. A. Izard, and P. Metcalfe: Med. Phys. 38 (2011) 4785.

8 A. S. Beddar, T. R. Mackie, and F. H. Attix: Phys. Med. Biol. 37 (1992) 1883.

9 A. S. Beddar, T. R. Mackie, and F. H. Attix: Phys. Med. Biol. 37 (1992) 1901.

10 D. E. Hyer, R. F. Fisher, and D. E. Hintenlang: Med. Phys. 36 (2009) 1711.

11 W. J. Yoo, S. H. Shin, D. Jeon, S. Hong, H. I. Sim, S. G. Kim, K. W. Jang, S. Cho, W. S. Youn, and B. Lee: Sensors 14 (2014) 6305. 